\title{
Longitudinal Study of the Transition From Healthy Aging to Alzheimer Disease
}

\author{
David K. Johnson, PhD; Martha Storandt, PhD; John C. Morris, MD; James E. Galvin, MD, MPH
}

Background: Detection of the earliest cognitive changes signifying Alzheimer disease is difficult.

Objective: To model the cognitive decline in preclinical Alzheimer disease.

Design: Longitudinal archival study comparing individuals who became demented during follow-up and people who remained nondemented on each of 4 cognitive factors: global, verbal memory, visuospatial, and working memory.

Setting: Alzheimer Disease Research Center, Washington University School of Medicine, St Louis, Missouri.

Participants: One hundred thirty-four individuals who became demented during follow-up and 310 who remained nondemented.

Main Oufcome Measures: Inflection point in longitudinal cognitive performance.

Results: The best-fitting model for each of the 4 factors in the stable group was linear, with a very slight downward trend on all but the Visuospatial factor. In contrast, a piecewise model with accelerated slope after a sharp inflection point provided the best fit for the group that progressed. The optimal inflection point for all 4 factors was prior to diagnosis of dementia: Global, 2 years; Verbal and Working Memory, 1 year; and Visuospatial, 3 years. These results were also obtained when data were limited to the subset $(n=44)$ with autopsy-confirmed Alzheimer disease.

Conclusions: There is a sharp inflection point followed by accelerating decline in multiple domains of cognition, not just memory, in the preclinical period in Alzheimer disease when there is insufficient cognitive decline to warrant clinical diagnosis using conventional criteria. Early change was seen in tests of visuospatial ability, most of which were speeded. Research into early detection of cognitive disorders using only episodic memory tasks may not be sensitive to all of the early manifestations of disease.

Arch Neurol. 2009;66(10):1254-1259
Author Affiliations: Department of Psychology, University of Kansas, Lawrence, Kansas City, Missouri (Dr Johnson); and Alzheimer Disease Research Center (Drs Storandt, Morris, and Galvin) and Departments of Psychology (Dr Storandt), Neurology (Drs Morris and Galvin), Pathology and Immunology (Dr Morris), Psychiatry (Dr Galvin), and Neurobiology (Dr Galvin), Washington University School of Medicine, St Louis, Missouri.

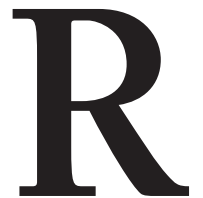

ECENT STUDIES HAVE FOcused on identifying the beginning of the transition from healthy aging to dementia. As new interventions become available, it will become important to identify the disease as early as possible. A piecewise regression analysis of a measure of episodic memory identified an inflection point 5 years before diagnosis in the Bronx Aging Study. ${ }^{1}$ A flat trajectory followed by decline beginning 7 years before diagnosis of dementia was reported for the same measure in the Baltimore Longitudinal Study of Aging, ${ }^{2}$ which also found decline in executive function that increased in rate 2 to 3 years before diagnosis. Episodic memory is not the only aspect of cognition that can be affected in preclinical Alzheimer disease (AD). ${ }^{3}$ Indeed, mild cognitive impairment, often thought to represent a transitional state between healthy cognitive ag- ing and $\mathrm{AD}$, is defined on the basis of deficits in cognitive domains in addition to memory. ${ }^{4}$

In this article we examine cognitive domains beyond episodic memory and executive function to test hypotheses about the existence of inflection points before clinical diagnosis of dementia. Based on the identification of common factor structures in cognitively healthy individuals and those with $\mathrm{AD},{ }^{5}$ we examined global mental ability and 3 specific cognitive domains (verbal memory, working memory, and visuospatial ability) through a long preclinical period as people developed dementia and compared them with those who remained cognitively healthy. Results from longitudinal studies ${ }^{6-9}$ support an early observation ${ }^{10}$ that the overall course of healthy aging is relatively stable compared with the cognitive decline, often precipitous, experienced by those who develop dementia. We sought to model 
Table 1. Characteristics of the Samples at Entry, Time of Dementia Diagnosis, and Last Assessment

\begin{tabular}{|c|c|c|c|}
\hline Variable & $\begin{array}{l}\text { Stable } \\
\text { Group } \\
(n=310)\end{array}$ & $\begin{array}{c}\text { Progressed } \\
\text { Group } \\
(n=134)\end{array}$ & $\begin{array}{c}\text { Autopsied } \\
\text { Group } \\
(n=44)\end{array}$ \\
\hline Assessments, No. & 1581 & 957 & 330 \\
\hline Age at entry, mean (SD), y & $74.4(8.6)$ & $80.4(8.9)$ & $83.6(9.2)$ \\
\hline $\begin{array}{l}\text { Age at diagnosis, mean } \\
\text { (SD), y }\end{array}$ & NA & 84.1 & $90.2(7.2)$ \\
\hline $\begin{array}{l}\text { Age at last assessment, } \\
\text { mean (SD), y }\end{array}$ & $79.6(8.5)$ & $88.2(8.2)$ & $92.2(6.3)$ \\
\hline Male sex, \% & 37 & 34 & 36 \\
\hline APOE4 carrier, \% & 28 & 27 & 18 \\
\hline Education, mean, y & 14.8 & 14.1 & 13.8 \\
\hline $\begin{array}{l}\text { SBT score at entry, } \\
\text { mean (SD) }\end{array}$ & $1.4(2.1)$ & $1.6(2.1)$ & $1.5(2.1)$ \\
\hline $\begin{array}{l}\text { SBT score at diagnosis, } \\
\text { mean (SD) }\end{array}$ & NA & $4.6(4.8)$ & $5.5(6.1)$ \\
\hline $\begin{array}{l}\text { SBT score at last } \\
\quad \text { assessment, mean (SD) }\end{array}$ & $1.2(1.9)$ & $7.1(7.0)$ & $9.9(8.7)$ \\
\hline CDR-SB at entry, mean (SD) & $0.05(0.2)$ & $0.17(0.3)$ & 0 \\
\hline $\begin{array}{l}\text { CDR-SB at diagnosis, } \\
\text { mean (SD) }\end{array}$ & NA & $1.5(1.8)$ & $1.7(2.4)$ \\
\hline $\begin{array}{l}\text { CDR-SB at last assessment, } \\
\text { mean (SD) }\end{array}$ & $0.03(0.1)$ & $3.3(3.7)$ & $4.5(5.1)$ \\
\hline
\end{tabular}

Abbreviations: APOE4, apolipoprotein E4; CDR-SB, Clinical Dementia Rating sum of boxes; NA, not applicable; SBT, Short Blessed Test.

that change to determine when the inflection occurs before dementia detection and the rate of change afterward for different cognitive domains and to validate these clinical observations in an autopsy-confirmed sample.

\section{METHODS}

\section{PARTICIPANTS}

Longitudinal archival data were examined from 444 volunteers initially aged 60 to 101 years enrolled in the Alzheimer Disease Research Center, Washington University School of Medicine, St Louis, Missouri, between October 1, 1979, and December 31, 2006 (Table 1). All the participants were clinically evaluated to be cognitively healthy (Clinical Dementia Rating ${ }^{11}$ $[C D R]=0)$ at the time of their first psychometric assessment and had at least 1 additional annual clinical evaluation through November 29, 2007. The Washington University Human Studies Committee approved all the procedures. Data from these participants have been used in other publications.

\section{CLINICAL EVALUATION}

Research-trained clinicians and nurses determined whether the participant was demented $(C D R>0)$ or not demented $(C D R=0)$ based on semistructured interviews with the participant and a knowledgeable collateral source (usually the spouse or an adult child), a health history, medication and depression inventories, an aphasia battery, and a neurologic examination of the participant. The diagnosis of dementia was based on a history of gradual onset and progressive cognitive decline that interfered with the person's ability to perform accustomed activities. The CDR has high interrater reliability, ${ }^{12}$ is sensitive to clinical progression, and is highly predictive (93\%) of autopsyconfirmed AD. ${ }^{13}$ Participants were seen by different physi-
Table 2. Initial Neuropsychological Performance for the Stable and Progressed Groups

\begin{tabular}{lcc|}
\hline \multirow{2}{*}{ Measure } & \multicolumn{2}{c}{ Score, Mean (SD) } \\
\cline { 2 - 3 } & Stable Group & Progressed Group \\
\hline Logical Memory & $8.87(2.91)$ & $7.39(2.93)$ \\
Associate Learning & $13.42(3.53)$ & $11.90(3.30)$ \\
Information & $20.60(4.43)$ & $18.94(5.04)$ \\
Boston Naming & $54.55(5.69)$ & $51.00(7.02)$ \\
Mental Control & $7.21(1.78)$ & $7.07(1.97)$ \\
Digit Span Forward & $6.55(1.25)$ & $6.40(1.15)$ \\
Digit Span Backward & $4.75(1.28)$ & $4.62(1.18)$ \\
Letter Fluency & $29.41(9.73)$ & $27.21(9.79)$ \\
Block Design & $30.05(8.63)$ & $26.86(7.02)$ \\
Digit Symbol & $45.67(11.53)$ & $26.86(7.02)$ \\
Trailmaking A, s & $40.94(19.88)$ & $51.21(21.82)$ \\
Benton, copy & $9.59(0.88)$ & $9.78(0.47)$ \\
& &
\end{tabular}

cians from year to year, and physicians did not have access to previous clinical evaluations or to previous or current psychometric test results.

\section{PSYCHOMETRIC ASSESSMENT}

The psychometric battery was administered to all the participants by trained psychometricians usually 1 to 2 weeks after the annual clinical assessment. The tests assessed a broad spectrum of abilities across multiple cognitive domains, including Logical Memory, Associate Learning, Mental Control, and Digit Span from the Wechsler Memory Scale (WMS) ${ }^{14}$; Information, Block Design, and Digit Symbol from the Wechsler Adult Intelligence Scale ${ }^{15}$; the Boston Naming Test ${ }^{16}$; Letter Fluency for $\mathrm{S}$ and $\mathrm{P}^{17}$; Trailmaking Test Part $\mathrm{A}^{18}$; and Form $\mathrm{D}$ (copy) of the Benton Visual Retention Test (Table 2). ${ }^{19}$ The raw scores from each test were converted to standard scores using the means and standard deviations from the initial assessment of the stable group (Table 2). Initial values for the group that progressed are included solely for descriptive purposes; recall that they were initially older, on average, than the stable group.

Based on confirmatory factor analyses of these measures cross-validated across demented and nondemented samples, ${ }^{5}$ we formed 4 factor scores for each person at each assessment. The Global factor included all 12 measures; it was uncorrelated with 3 specific factors, which each included 4 measures. The measures on the Verbal Memory factor were Logical Memory, Associate Learning, Information, and Boston Naming. The 4 measures on the Working Memory and Executive Function factor were Mental Control, Digit Span Forward and Backward, and Letter Fluency. The Visuospatial factor included Block Design, Digit Symbol, Trailmaking A, and Benton (copy). A prorated factor score was computed if 1 or 2 values were missing for the Global factor and if 1 value was missing for the specific factors; otherwise, the factor value for that assessment for that person was excluded from the analyses.

\section{NEUROPATHOLOGIC ASSESSMENT}

All the brains were examined according to a standard protocol. ${ }^{20}$ After fixation in neutral-buffered $10 \%$ formalin, tissue blocks were obtained from 30 brain regions. Sections $(6 \mu \mathrm{m})$ from paraffin-embedded tissue blocks were stained with hematoxylin-eosin, Gallyas and modified Bielschowsky silver stains, and immunohistochemical methods. Histologic criteria for $\mathrm{AD}$ were based on the quantification of diffuse and neuritic amyloid deposition in 5 cortical regions with $10-\mathrm{mm}^{2} \mathrm{mi}-$ 
croscopic fields in each region and the National Institute on Aging-Reagan ${ }^{21}$ neuropathologic probability estimates of AD. The 2 sets of criteria have near-complete agreement for intermediate and high probability of AD.

\section{STATISTICAL ANALYSES}

Cross-sectional comparisons of quantitative demographic variables (age and education) in the 2 groups (stable vs progressed) were made using $t$ tests for independent groups; the $\chi^{2}$ test was used for categorical variables. A multistep longitudinal modeling procedure was used for each of the 4 factors. All longitudinal analyses were conducted using random coefficient models (SAS v9.1.3, PROC MIXED; SAS Institute Inc, Cary, North Carolina) and included the covariates of age and education.

To determine the best form of a factor score's trajectory through time for each group (stable and progressed), we used $\chi^{2}$ tests for $-2 \log$ likelihood ratios ( -2 LLs) for nested models of increasing complexity of the slope across time (linear, quadratic, linear piecewise, linear piecewise optimized, and quadratic piecewise); simpler models are nested in more complex models. Model comparisons used $\chi^{2}$ tests of deviance scores beginning with the simple linear model. Deviance scores equal the difference between the -2LL of a simpler model and a more complex one $\left(\Delta \chi^{2}\right)$.

The linear piecewise change model specified a point of inflection and tested whether rates of cognitive decline differed before and after that point. ${ }^{22}$ To determine the optimum placement of the change point in the piecewise model, we tested inflection points from 1 to 6 years before the last assessment for the stable group. For those who progressed, we tested for an inflection point at the time of diagnosis of dementia and from 4 years before diagnosis to 2 years after diagnosis ( $>50$ observations at each selected time point). The quadratic piecewise model added a quadratic term for the postinflection time variable using the optimal inflection point.

We conducted additional analyses to determine whether instead of a quadratic function after inflection there was acceleration in the rate of progression (ie, a change in the slope of the slopes across time). Technically, this was not a test of a nested model. First, we used the optimal piecewise model to predict the factor scores at each time of assessment for each person (best linear unbiased predictor). Using these latent values from each time of assessment rather than observed values, we calculated latent difference scores (LDSs) for each person. The LDS equals the difference between the predicted values at 2 adjacent times of assessment $\left(\hat{\mathrm{Y}}_{\mathrm{T} 1}-\hat{\mathrm{Y}}_{\mathrm{T} 2}, \hat{\mathrm{Y}}_{\mathrm{T} 2}-\hat{\mathrm{Y}}_{\mathrm{T} 3}, \hat{\mathrm{Y}}_{\mathrm{T} 3}-\hat{\mathrm{Y}}_{\mathrm{T} 4}\right.$, and so forth) beginning with the difference of the predicted value at the optimized inflection point $\left(\hat{\mathrm{Y}}_{\mathrm{T} 1}\right)$ and the next assessment thereafter $\left(\hat{\mathrm{Y}}_{\mathrm{T} 2}\right)$.

Then we tested a linear mixed model using the LDS as the dependent variable to determine whether the slope of the LDS values (ie, the slope of the slopes) changed across time. The acceleration coefficient tested herein is a 2-stage regression analogue to other acceleration models derived from structural equation modeling ${ }^{23}$ and the functional equivalent of acceleration in kinematics (ie, the second derivative of position/intercept). Although this method of measurement may slightly attenuate true acceleration values because of the tendency for latent values to "shrink" in the presence of missing data, ${ }^{24}$ its computation is robust, is straightforward, and can be estimated without multiple imputation of missing data. It yields results consistent with simultaneous models. ${ }^{25} \mathrm{~A}$ simultaneous model that approximates slopes and acceleration was not attempted because we did not know the functional form of the data (the primary aim of this investigation).

After determining the optimal models within each group, slope estimates were based on a mixed model for each factor that included covariates, effects for group (stable vs progressed), time (before and after inflection), and group $\times$ time interactions. Acceleration coefficients for the group that progressed were estimated from the second stage of the LDS model and were added to slope coefficients after the point of inflection. All analyses were repeated for the subset of the group that progressed that had autopsy confirmation of AD.

\section{RESULTS}

\section{SAMPLE CHARACTERISTICS}

All the participants were not demented $(C D R=0)$ at entry and either remained CDR $=0$ (stable) throughout follow-up $(n=310,37 \%$ men) or progressed to CDR $>0$ $(\mathrm{n}=134,34 \%$ men) with a clinical diagnosis of uncertain dementia $(C D R=0.5)$ or dementia of the Alzheimer type $(C D R \geq 0.5)$ by the time of their last evaluation (Table 1). Participants who progressed and whose clinical diagnosis was non-AD dementia (eg, vascular dementia associated with Parkinson disease) were excluded. Individuals who came to autopsy with a clinical diagnosis of dementia of the Alzheimer type but who had another dementia abnormality were also excluded $(n=14)$. Maximum follow-up was 25.7 years (mean [SD], 5.9 [5.3] years). The stable group was slightly more educated (mean, 14.8 years) than the group that progressed (mean, 14.1 years). As might be expected given that AD is age associated, those who progressed were older at entry (mean [SD], 80.4 [8.9] years) than those whose performance remained stable (mean [SD], 74.4 [8.6] years). Apolipoprotein E4 status did not differ between the stable ( $28 \%$ carriers) and progressed ( $27 \%$ carriers) groups. Autopsy confirmation of a diagnosis of AD was available for a subset ( $n=44,36 \%$ men) of the group that progressed. At the time of progression, they were older (mean age, 90.2 years) than the rest of the group (mean age, 84.1 years), although their mean educational level (13.8 years) was comparable with that of those without autopsy (14.1 years).

\section{NONDEMENTED AGING}

The linear regression model provided the best fit for each factor in the stable group. The -2LL values were as follows: Global, 4255.5; Verbal Memory, 3045.3; Visuospatial, 2796.7; and Working Memory, 5912.5. More complex models did not improve fit $\left(\Delta \chi^{2}>14.4\right.$ for all, $P>.05)$.

\section{CHARACTERIZING PRECLINICAL AD: INFLECTION POINTS}

In the progressed group, the linear model provided adequate fit for all 4 factors (-2LL values) (Table 3). Fit was not improved using a quadratic model for any factor $(P>.05$ for all), although it was improved using a piecewise model with an inflection point at the time of diagnosis. The piecewise model fit was improved by moving the inflection point before diagnosis $(P<.001)$ (Table 3). The optimal inflection point varied for the 4 factors: 2 years before clinical diagnosis for the Global 
Table 3. -2LL Values for Each Cognitive Factor for Each Model and Significant Maximum Likelihood Deviance Tests $\left(\Delta \chi^{2}\right)^{\mathrm{a}}$ for the Group That Progressed

\begin{tabular}{|c|c|c|c|c|c|c|c|c|c|}
\hline \multirow[b]{2}{*}{ Model } & \multirow[b]{2}{*}{$d f$} & \multicolumn{2}{|c|}{ Global Factor } & \multicolumn{2}{|c|}{ Verbal Memory Factor } & \multicolumn{2}{|c|}{ Visuospatial Factor } & \multicolumn{2}{|c|}{ Working Memory Factor } \\
\hline & & $-2 \mathrm{LL}$ & $\Delta \boldsymbol{x}^{2}$ & $-2 \mathrm{LL}$ & $\Delta \boldsymbol{x}^{2}$ & $-2 \mathrm{LL}$ & $\Delta x^{2}$ & $-2 \mathrm{LL}$ & $\Delta \chi^{2}$ \\
\hline Simple linear & 8 & 2750.8 & NA & 2277.7 & NA & 1938.8 & NA & 3737.8 & NA \\
\hline Quadratic & 9 & 3019.5 & 268.7 & 2277.7 & 0 & 1938.8 & 0 & 3737.8 & 0 \\
\hline \multicolumn{10}{|l|}{ Piecewise linear } \\
\hline At diagnosis & 9 & 2705.1 & -45.7 & 2255.9 & -21.8 & 1939.6 & -44.2 & 3690.1 & -47.7 \\
\hline Optimized ${ }^{\mathrm{b}}$ & 9 & 2690.3 & -14.8 & 2243.5 & -12.4 & 1910.3 & -29.3 & 3676.0 & -20.1 \\
\hline Piecewise quadratic ${ }^{c}$ & 10 & 2689.5 & -0.8 & 2243.9 & 0.4 & 1910.3 & 0 & 3675.6 & -0.4 \\
\hline
\end{tabular}

Abbreviations: -2LL, -2 log likelihood ratio (smaller values indicate better fit); NA, not applicable.

a 1 df for deviance tests.

b The optimal inflection point was 2 years before diagnosis for the Global factor, 3 years before diagnosis for the Visuospatial factor, and 1 year before diagnosis for the Verbal and Working Memory factors.

${ }^{\complement}$ Quadratic term after the inflection point.

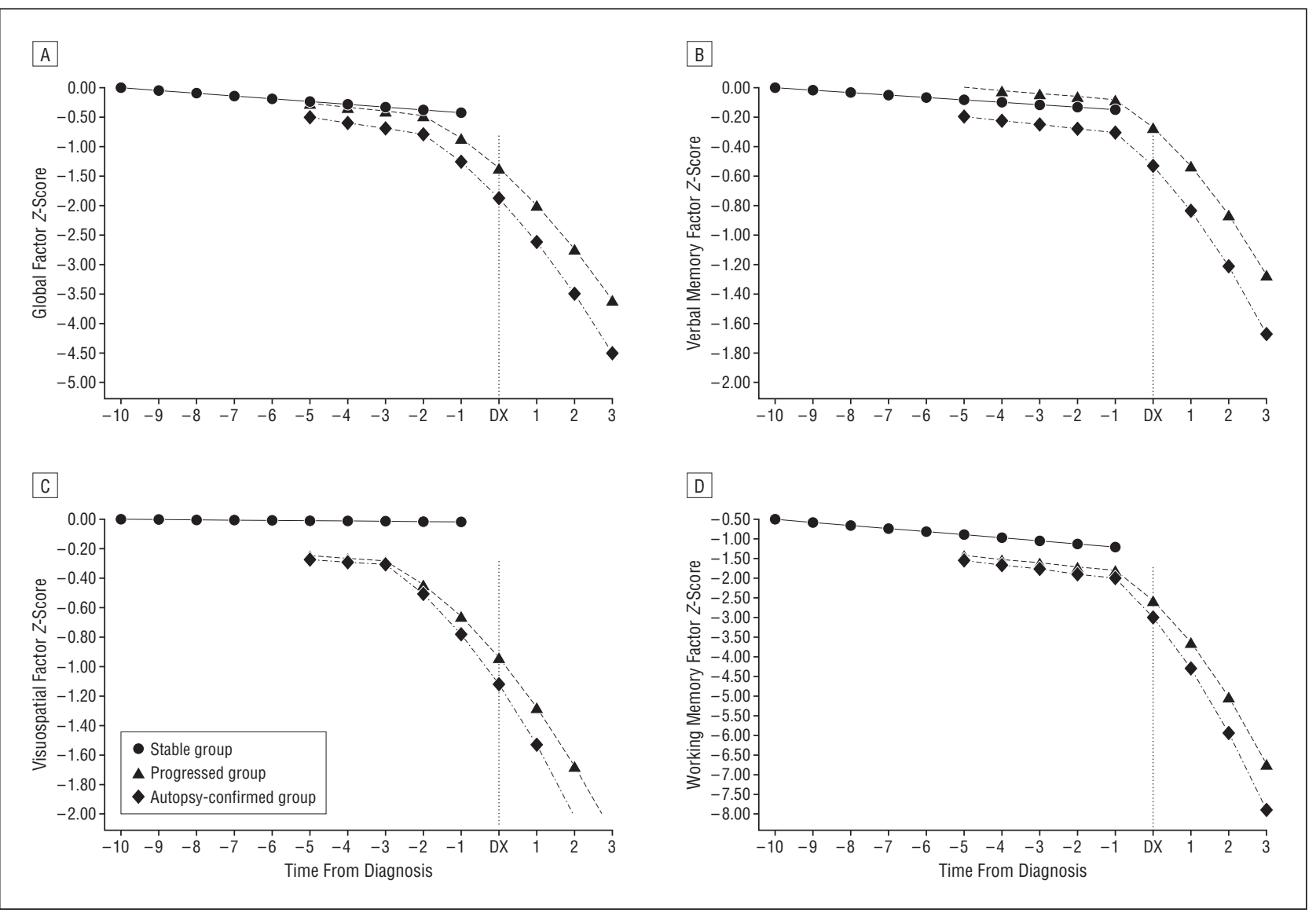

Figure. Longitudinal course of the stable, progressed, and autopsy-confirmed Alzheimer disease (AD) groups before and after diagnosis of AD (DX) on Global factor (A), the Verbal Memory factor (B), the Visuospatial factor (C), and the Working Memory factor (D). All available data were used for the analysis, but the plotted values for the stable and progressed groups include at least 50 observations per time point per group.

factor, 1 year before diagnosis for the Verbal and Working Memory factors, and 3 years before diagnosis for the Visuospatial factor (Figure).

The Verbal Memory factor included episodic and semantic memory measures. An inflection point for a single measure of episodic memory occurred substantially longer than 1 year before diagnosis in previous studies. ${ }^{1,2}$ Therefore, we also examined each of the 2 measures of episodic memory independently. The inflection point occurred 4 years before diagnosis for WMS Associate
Learning and 2 years before diagnosis for WMS Logical Memory.

The fit of the piecewise model did not improve with the addition of a quadratic term after the optimal inflection point $(P>.05$ for all) (Table 3$)$. There was, however, a significant increase in the rate of decline after the inflection point for all 4 factors using the LDS models of acceleration $(t>12.10$ for all, $P<.001)$. Thus, the optimal model in the group that progressed was piecewise, with linear slope before inflection and accelerated slope

$$
\text { (REPRINTED) ARCH NEUROL/VOL } 66 \text { (NO. 10), OCT } 2009 \text { WWW.ARCHNEUROL.COM }
$$




\begin{tabular}{|c|c|c|c|}
\hline \multirow[b]{2}{*}{ Factor and Group } & \multirow{2}{*}{$\begin{array}{c}\begin{array}{c}\text { Before } \\
\text { Inflection }\end{array} \\
\begin{array}{|c|}\text { Slope } \\
\end{array}\end{array}$} & \multicolumn{2}{|c|}{$\begin{array}{c}\text { After } \\
\text { Inflection }\end{array}$} \\
\hline & & Slope & Acceleration \\
\hline \multicolumn{4}{|l|}{ Global factor } \\
\hline Stable & $-0.05(0.01)$ & NA & NA \\
\hline Progressed & $-0.07(0.02)$ & $-0.32(0.02)$ & $-0.06(0.003)$ \\
\hline AD confirmed & $-0.10(0.03)$ & $-0.40(0.04)$ & $-0.07(0.004)$ \\
\hline \multicolumn{4}{|c|}{ Verbal Memory factor } \\
\hline Stable & $-0.02(0.01)$ & NA & NA \\
\hline Progressed & $-0.02(0.01)$ & $-0.15(0.02)$ & $-0.04(0.001)$ \\
\hline AD confirmed & $-0.03(0.02)$ & $-0.19(0.03)$ & $-0.04(0.002)$ \\
\hline \multicolumn{4}{|l|}{ Visuospatial factor } \\
\hline Stable & $0.00(0.01)$ & NA & NA \\
\hline Progressed & $-0.02(0.01)$ & $-0.13(0.01)$ & $-0.03(0.001)$ \\
\hline AD confirmed & $-0.02(0.02)$ & $-0.17(0.02)$ & $-0.04(0.002)$ \\
\hline \multicolumn{4}{|c|}{ Working Memory factor } \\
\hline Stable & $-0.08(0.02)$ & NA & NA \\
\hline Progressed & $-0.10(0.03)$ & $-0.66(0.04)$ & $-0.17(0.01)$ \\
\hline AD confirmed & $-0.12(0.04)$ & $-0.89(0.06)$ & $-0.17(0.01)$ \\
\hline
\end{tabular}

Abbreviations: AD, Alzheimer disease; NA, not applicable.

${ }^{a}$ Data are given as estimate (SE). Slope coefficients were estimated using the piecewise change model of raw data. Acceleration coefficients were estimated using latent difference score regression, in which intercepts were equivalent to piecewise change slope estimates $( \pm 0.02 \mathrm{U})$.

bThe optimal inflection point was 2 years before diagnosis for the Global factor, 1 year before diagnosis for the Verbal and Working Memory factors, and 3 years before diagnosis for the Visuospatial factor.

afterward. The same was true when the data were limited to those who progressed and had autopsy confirmation of $\mathrm{AD}$, including the placement of the optimal inflection point for each factor.

\section{CHARACTERIZING PRECLINICAL AD: SLOPE AND ACCELERATION ESTIMATES}

Estimates and their standard errors are given in Table 4. Change in performance in all 4 factors is demonstrated in the Figure. The stable and progressed groups shared similar preinflection trajectories; the group $\times$ time (before inflection) interactions were not significant for any of the 4 factors $(P>.19$ for all). There was a significant downward linear trend in global cognitive abilities and in verbal and working memory $(P<.01)$; however, no longitudinal decline was detected in the Visuospatial factor $(P=.29)$ (Figure). Results were similar when data from the group that progressed were limited to individuals with autopsy-confirmed AD.

A different pattern of longitudinal cognitive performance was seen in the group that progressed to dementia. Compared with preinflection slopes, they had steeper downward slopes after inflection $(P<.001)$, and the rate of decline accelerated with time (Table 4 and Figure). The greatest preclinical slope change was in the Working Memory factor (slope $=-0.66$, acceleration $=-0.17$ ) beginning 1 year before dementia diagnosis followed by the Global factor (slope $=-0.32$, acceleration $=-0.06$ ). Postinflection slopes and accelerations were similar for the Verbal Memory and Visuospatial factors. Slopes were steeper when the sample that progressed was restricted to those with autopsy-confirmed $\mathrm{AD}$, but rates of acceleration were comparable with those for the total group that progressed.

\section{COMMENT}

We demonstrate models of preclinical decline in a wellcharacterized longitudinal sample with inflection points in cognitive performance occurring several years before clinical diagnosis of dementia. It is apparent from these models that there is a clear turning point in the transition from normal aging to preclinical AD. A novel finding was that visuospatial abilities demonstrated an inflection point 3 years before clinical diagnosis. This decline on tests that were primarily speeded represented a sharp departure from the previous longitudinal pattern of these initially nondemented individuals, which was similar to that of those who did not become demented. Global cognitive abilities followed decline in visuospatial ability during the next year. Inflection points in the Verbal and Working Memory factors were not seen until 1 year before clinical diagnosis. The delayed inflection point for Verbal Memory probably results from the combination of episodic and semantic memory measures on one factor. If sufficient measures of each type of memory were available to form separate factors, an earlier inflection point for episodic memory would probably emerge based on the results obtained for the 2 individual measures of episodic memory.

The rate of decline accelerated after the downward course began. This was true for all 4 factors but was most apparent for Working Memory. Of course, the estimated rates of decline and acceleration depend on the tests administered, their level of difficulty, and floor and ceiling effects. For example, 3 tests in the battery (the Boston Naming Test, the copy version of the Benton Visual Retention Test, and the WMS Mental Control) have ceiling effects in the preinflection period in nondemented older adults. We were limited to these archival data from a battery that was originally constructed in 1979 for a study of mild dementia, and it does not contain more modern measurements of working memory.

The number of years before diagnosis of dementia that the inflection point occurs in the longitudinal course depends on the method of diagnosis and on the characteristics of the cognitive tests. The period will be longer if one relies on test norms, particularly if the sample is at a high level of function initially, than if one relies on collateral source reports of change from previous levels of function, captured by the CDR. Furthermore, inclusion of people in the preclinical stage in nondemented samples overestimates decline in cognitive ability traditionally attributed solely to age. ${ }^{26}$ This makes it more difficult to detect beginning dementia using conventional norms based on these contaminated samples.

A great strength of this study is the replication of the pattern of the longitudinal results observed in the larger sample that progressed in the subset with autopsyconfirmed AD. Although the rates of decline were somewhat steeper in the autopsied subset, the rates of acceleration were the same. The rate of progression in $\mathrm{AD}$ is 
highly variable ${ }^{27}$; perhaps those with autopsy-confirmed AD were individuals who progressed more rapidly. Another possibility is that the progressed group may contain individuals who do not have AD and, therefore, do not follow the same pattern of decline.

There are several implications of this study. Some of the earliest signs of preclinical disease may occur on tests of visuospatial and speeded psychomotor skills. Furthermore, the greatest rate of preclinical decline may occur on executive and attention tasks. These findings suggest that research into early detection of cognitive disorders using only episodic memory tasks, such as word lists or paragraph recall, may not be sensitive to either all of the earliest manifestations of disease or the most rapidly changing domain. Furthermore, the preclinical downward course comes after an inflection point. Before that point, the longitudinal course of those who did and did not develop AD was the same. In summary, converging longitudinal evidence suggests that after a sharp departure from the relatively flat course of normal aging there is a preclinical period in AD with insufficient cognitive decline to warrant clinical diagnosis using conventional criteria but that can be seen with longitudinal data from multiple domains of cognition and not just memory.

Accepted for Publication: February 25, 2009.

Correspondence: James E. Galvin, MD, MPH, Alzheimer Disease Research Center, Washington University School of Medicine, 4488 Forest Park, Ste 130, St Louis, MO63108(galvinj@neuro.wustl.edu).

Author Contributions: All authors had full access to all the data used in this study. Drs Johnson and Galvin take full responsibility for the integrity of the data and the accuracy of the data analysis. Study concept and design: Johnson, Storandt, and Galvin. Acquisition of data: Storandt, Morris, and Galvin. Analysis and interpretation of data: Johnson, Storandt, and Galvin. Drafting of the manuscript: Johnson, Storandt, and Galvin. Critical revision of the manuscript for important intellectual content: Johnson, Storandt, Morris, and Galvin. Statistical analysis: Johnson and Storandt. Obtained funding: Storandt and Morris. Administrative, technical, and material support: Galvin. Study supervision: Morris and Galvin.

Financial Disclosure: None reported.

Funding/Support: This study was supported by grants P01 AG03991, P50 AG05681, P01 AG026276 (Dr Morris), and K08 AG20764 (Dr Galvin) from the National Institute on Aging, National Institutes of Health.

Additional Contributions: We thank the Clinical and Neuropathology Cores of the Washington University Alzheimer Disease Research Center for the clinical, cognitive, and postmortem assessments and the Genetics Core for the apolipoprotein E genotype data.

\section{REFERENCES}

1. Hall CB, Lipton RB, Sliwinski M, Stewart WF. A change point model for estimating the onset of cognitive decline in preclinical Alzheimer's disease. Stat Med. 2000;19(11-12):1555-1566.
2. Grober E, Hall CB, Lipton RB, Zonderman AB, Resnick SM, Kawas C. Memory impairment, executive dysfunction, and intellectual decline in preclinical Alzheimer's disease. J Int Neuropsychol Soc. 2008;14(2):266-278.

3. Twamley EW, Ropacki SA, Bondi MW. Neuropsychological and neuroimaging changes in preclinical Alzheimer's disease. J Int Neuropsychol Soc. 2006;12 (5):707-735.

4. Winblad B, Palmer K, Kivipelto M, et al. Mild cognitive impairment: beyond controversies, towards a consensus: report of the International Working Group on Mild Cognitive Impairment. J Intern Med. 2004;256(3):240-246.

5. Johnson DK, Storandt M, Morris JC, Langford ZD, Galvin JE. Cognitive profiles in dementia: Alzheimer disease versus nondemented healthy brain aging. Neurology. 2008;71(22):1783-1789.

6. Howieson DB, Dame A, Camicioli R, Sexton G, Payami H, Kaye JA. Cognitive markers preceding Alzheimer's dementia in the healthy oldest old. J Am Geriatr Soc. 1997;45(5):584-589.

7. Rubin EH, Storandt M, Miller JP, et al. A prospective study of cognitive function and onset of dementia in cognitively healthy elders. Arch Neurol. 1998;55(3): 395-401.

8. Chen P, Ratcliff G, Belle SH, Cauley JA, DeKosky ST, Ganguli M. Patterns of cognitive decline in presymptomatic Alzheimer disease. Arch Gen Psychiatry. 2001; 58(9):853-858.

9. Galvin JE, Powlishta KK, Wilkins K, et al. Predictors of preclinical Alzheimer disease and dementia: a clinicopathologic study. Arch Neurol. 2005;62(5):758765.

10. Granik S. Psychological test functioning. In: Granick S, Patterson RD, eds. Human Aging. 2nd ed. Rockville, MD: US Dept of Health, Education, and Welfare; 1971. DHEW publication (HSM) 71-9037.

11. Morris JC. The Clinical Dementia Rating (CDR): current version and scoring rules. Neurology. 1993;43(11):2412-2414.

12. Burke WJ, Miller JP, Rubin EH, et al. Reliability of the Washington University Clinical Dementia Rating. Arch Neurol. 1988;45(1):31-32.

13. Berg L, McKeel DW Jr, Miller JP, et al. Clinicopathologic studies in cognitively healthy aging and Alzheimer disease: relation of histologic markers to dementia severity, age, sex, and apolipoprotein E genotype. Arch Neurol. 1998;55(3): 326-335.

14. Wechsler D, Stone CP. Manual: Wechsler Memory Scale. New York, NY: Psychological Corp; 1973.

15. Wechsler D. Manual: Wechsler Adult Intelligence Scale. New York, NY: Psychological Corp; 1955.

16. Goodglass H, Kaplan E. The Assessment of Aphasia and Related Disorders. 2nd ed. Philadelphia, PA: Lea \& Febiger; 1983.

17. Thurstone LL, Thurstone LG. Examiner Manual for the SRA Primary Mental Abilities Test. Chicago, IL: Science Research Associates; 1949.

18. Armitage SG. An analysis of certain psychological tests used in the evaluation of brain injury. Psychol Monogr. 1946;60:1-48.

19. Benton AL. The Revised Visual Retention Test: Clinical and Experimental Applications. New York, NY: Psychological Corp; 1963.

20. McKeel DW Jr, Ball MJ, Price JL, et al. Interlaboratory histopathologic assessment of Alzheimer neuropathology: different methodologies yield comparable diagnostic results. Alzheimer Dis Assoc Disord. 1993;7(3):136-151.

21. National Institute on Aging, Reagan Institute Working Group on Diagnostic Criteria for the Neuropathological Assessment of Alzheimer's Disease. Consensus recommendations for the postmortem diagnosis of Alzheimer's disease. Neurobiol Aging. 1997;18(4)(suppl):S1-S2.

22. Fitzmaurice GM, Laird NM, Ware JH. Applied Longitudinal Analysis. Hoboken, NJ: John Wiley \& Sons; 2004

23. Hamagami F, McArdle JJ. Dynamic extensions of latent difference score models. In: Boker SM, Wenger MJ, eds. Notre Dame Series on Quantitative Methodology: Data Analytic Techniques for Dynamical Systems. Mahwah, NJ: Lawrence Erlbaum Associates; 2007.

24. Efron B, Morris C. Stein's paradox in statistics. Sci Am. 1977;236:119-127.

25. Chou CP, Bentler PM, Pentz MAA. Two-stage approach to multilevel structural equation models: application to longitudinal data. In: Little TD, Schnabel KU, Baumert J, eds. Modeling Longitudinal and Multilevel Data. Mahwah, NJ: Lawrence Erlbaum Associates; 2000:33-49

26. Sliwinski M, Lipton RB, Buschke $H$, Stewart W. The effects of preclinical dementia on estimates of normal cognitive functioning in aging. J Gerontol B Psychol Sci Soc Sci. 1996;51(4):P217-P225.

27. Storandt M, Grant EA, Miller JP, Morris JC. Rates of progression in mild cognitive impairment and early Alzheimer disease. Neurology. 2002;59(7):10341041 